\title{
Importância do conhecimento sobre sinais e sintomas da pré-eclâmpsia para implementação dos cuidados de Enfermagem
}

\author{
Importance of knowledge about signs and symptoms of pre-eclampsia for Nursing care \\ implementation
}
Importancia del conocimiento sobre signos y síntomas de la pre eclampsia para implementación de los cuidados de enfermería

Rosane da Silva Santana ${ }^{1}$, Ayla Cristina Rodrigues Ramos da Costa ${ }^{2}$,Francisco Lucas de Lima Fontes $^{2 *}$, Francisco Rafael de Carvalho², Fabrícia Ferreira de Moura ${ }^{3}$, Jackson Menezes Duarte ${ }^{2}$, Jorge Ferreira da $\mathrm{Cruz}^{2}$, Jakson de Oliveira Gaia ${ }^{2}$, Thainara Dias da Silva ${ }^{2}$, Jancielle Silva Santos ${ }^{4}$, Ana Beatriz Brito Alencar ${ }^{2}$, Antônia Vanessa Leal de Sousa ${ }^{2}$, Andressa de Oliveira Lima ${ }^{2}$, Marjory Ribeiro Botelho Veloso², Aline dos Santos Silva².

\begin{abstract}
RESUMO
Objetivo: Analisar a importância do conhecimento sobre sinais e sintomas da pré-eclâmpsia para implementação dos cuidados de Enfermagem. Métodos: Estudo descritivo com abordagem qualitativa realizado com cinco enfermeiras de uma maternidade privada de Teresina, capital do Piauí, nos meses de outubro e novembro de 2017 após aprovação do Comitê de Ética em Pesquisa. Resultados: O cumprimento da prescrição medicamentosa e aferição dos sinais vitais, em especial pressão arterial, foram os cuidados comumente implementados. Também verificou-se que nenhuma das entrevistadas mencionou cuidados com a vitalidade fetal, priorizando-se apenas a monitorização da mãe; o que evidencia fragilidade da assistência realizada no quesito binômio mãe-filho. Conclusão: Conhecer as condutas de Enfermagem implementadas ao seu tratamento é essencial para a garantia de qualidade do atendimento ofertado. Salienta-se uma falha primária na assistência identificada neste estudo, pois muitas mulheres só descobrem a patologia no momento de internação, o que gera inúmeros desconfortos emocionais para a gestante que já passa por alterações hormonais que afetam seus sentimentos.
\end{abstract}

Palavras-chave: Complicações na gravidez, Pré-eclâmpsia, Assistência de Enfermagem.

\begin{abstract}
Objective: To analyze the importance of knowledge about signs and symptoms of preeclampsia for the implementation of nursing care. Methods: Descriptive study with a qualitative approach carried out with five nurses from a private maternity hospital in Teresina, capital of Piauí, in October and November 2017, after approval by the Research Ethics Committee. Results: The compliance with the prescription and measurement of vital signs, especially blood pressure, were the usual care. It was also verified that not one of the interviewees mentioned care with fetal vitality, giving priority to monitoring the mother, which shows the fragility of the care performed in the binomial mother-child relationship. Conclusion: Knowing the Nursing conducts implemented to their treatment is essential for the quality assurance of the offered care. A primary flaw in the care identified in this study is highlighted, in which many women only discover the pathology at the moment of hospitalization, which generates innumerable emotional discomforts for the pregnant woman who already undergoes hormonal changes that affect her feelings.
\end{abstract}

Keywords: Pregnancy complications, Pre-eclampsia, Nursing care.

1 Universidade Federal do Ceará. Fortaleza, Ceará, Brasil.

${ }^{2}$ Centro Universitário Maurício de Nassau (UNINASSAU) - Campus Redenção. Teresina, Piauí, Brasil.

*E-mail: lucasfontesenf@hotmail.com

3Universidade Federal do Piauí. Picos, Piauí, Brasil.

${ }^{4}$ Centro Universitário Santo Agostinho. Teresina, Piauí, Brasil.

SUBMETIDO EM: 8/2019

ACEITO EM: 9/2019

PUBLICADO EM: 9/2019

REAS/EJCH |Vol.11(15) | e1425 | DOI: https://doi.org/10.25248/reas.e1425.2019 Página 1 de 6 


\section{RESUMEN}

Objetivo: Analizar la importancia del conocimiento sobre signos y síntomas de la preeclampsia para implementación de los cuidados de Enfermería. Métodos: Estudio descriptivo con abordaje cualitativo realizado con cinco enfermeras de una maternidad privada de Teresina, capital de Piauí, en los meses de octubre y noviembre de 2017 tras la aprobación del Comité de Ética en Investigación. Resultados: El cumplimiento de la prescripción medicamentosa y la medida de los signos vitales, en especial presión arterial, fueron los cuidados comúnmente implementados. También se verificó que ninguna de las entrevistadas mencionó cuidados con la vitalidad fetal, priorizándose apenas la monitorización de la madre, lo que evidencia fragilidad de la asistencia realizada en el aspecto binomio madre-hijo. Conclusión: Conocer las conductas de Enfermería implementadas a su tratamiento es esencial para la garantía de calidad de la atención ofrecida. Se destaca una falla primaria en la asistencia identificada en este estudio, pues muchas mujeres sólo descubren la patología en el momento de internación, lo que genera innumerables molestias emocionales para la gestante que ya pasa por alteraciones hormonales que afectan sus sentimientos.

Palabras-clave: Complicaciones del embarazo, Preeclampsia, Atención de Enfermería.

\section{INTRODUÇÃO}

A pré-eclâmpsia é um transtorno que pode surgir na gravidez após a vigésima semana gestacional, durante o parto e até 48 horas pós-parto. Ela é definida por aumento da pressão arterial superior a 140x90 $\mathrm{mmHg}$, presença de proteínas na urina e anasarca por conta da retenção de líquidos (FERREIRA GD et al., 2013).

A Organização Mundial de Saúde (OMS) evidencia que de $80 \%$ das mulheres são levadas a óbito em decorrência das complicações da pré-eclâmpsia durante a gravidez ou parto. No Brasil encontra-se em segundo lugar nas causas de mortes maternas, atingindo cerca de 5 a 17\% das gestantes (SAMPAIO TAF et al., 2013; OLIVEIRA ACM et al., 2016).

Durante o período gestacional essa complicação pode relacionar-se a condições socioeconômicas e clínicas desfavoráveis como escolaridade insuficiente, baixa renda familiar, gestações de risco e circunstâncias nutricionais e obstétricas inadequadas (MASSUCATTI LA et al., 2012).

Dentre as principais manifestações clínicas apresentadas na pré-eclâmpsia encontram-se náuseas, vômitos, dor em região epigástrica que irradia para membros superiores, cefaleia, alterações visuais (visão turva), hiperreflexia, taquipneia e ansiedade. Contudo, em algumas gestantes a doença pode evoluir de forma silenciosa (BRITO KKG et al., 2015).

As recomendações oferecidas a essas gestantes são orientações quanto ao repouso, aferição da pressão arterial ao longo do dia, controle rigoroso do peso e da diurese e verificação dos movimentos fetais, além de acompanhamento clínico rigoroso oferecido por equipe multidisciplinar (AGUIAR MIF et al., 2010).

Partindo dessa prerrogativa, é fundamental a atuação do enfermeiro dentro da equipe multidisciplinar na prestação de assistência adequada e eficaz à gestante com essa complicação. A equipe de saúde deve estar preparada para enfrentar quaisquer fatores que possam afetar adversamente a gravidez (SOUZA ALS et al., 2011).

Compreende-se, portanto, que o enfermeiro possui formação e competência para aplicar seus conhecimentos técnicos e científicos na prática assistencial, visando um cuidado singular e coerente de acordo com as condições apresentadas pela paciente (MOURA MDR et al., 2011).

Diante do explanado, o objetivo do presente estudo foi analisar a importância do conhecimento sobre sinais e sintomas da pré-eclâmpsia para implementação dos cuidados de Enfermagem.

\section{MÉTODOS}

Trata-se de um estudo descritivo com abordagem qualitativa, realizado nos setores de atendimento às urgências obstétricas, enfermarias e apartamentos de uma maternidade privada de Teresina, capital do Piauí, nos meses de outubro e novembro de 2017. 
Participaram do estudo cinco dos seis enfermeiros que prestavam assistência nesses setores. Foram incluídos enfermeiros diaristas e plantonistas que trabalhavam há pelo menos um ano nos locais destinados ao tratamento de patologias gravídicas. E excluídos, aqueles que não atuavam na assistência direta a essas gestantes ou que estavam de férias ou licença no período da coleta de dados.

Para coleta de dados utilizou-se a técnica de entrevista, por meio de um roteiro semiestruturado. Como garantia à segurança, ao sigilo e ao anonimato dos entrevistados, os participantes foram identificados com a letra $E$ seguido de um algarismo arábico na sequência de realização das entrevistas, de modo que todos os sujeitos da pesquisa puderam certificar-se de que suas identidades permaneceriam anônimas.

Para que nenhuma informação relevante fosse perdida ou esquecida, foi utilizado como recurso um aparelho gravador de voz, objetivando garantir a autenticidade dos depoimentos representados pela fala dos entrevistados.

A análise dos depoimentos foi desenvolvida por meio da técnica de "Análise de Conteúdo". Essa análise consiste em três etapas: a pré-análise, em que será realizada a leitura flutuante das entrevistas a fim de sistematizar as ideias iniciais, em seguida, exploração do material: identificando os núcleos de sentido das falas agrupando as ideias que se relacionarem e por fim o tratamento dos dados obtidos (MINAYO MCS, 2014).

O estudo foi aprovado pelo Comitê de Ética em Pesquisa da Faculdade de Ciências e Tecnologia do Maranhão (FACEMA), com no de CAAE 74232017.8.0000.8007 e parecer oㅜ 2.313.070. Cada entrevista foi conduzida somente após esclarecimento dos objetivos da pesquisa e consentimento do participante, mediante assinatura do Termo de Consentimento Livre e Esclarecido.

\section{RESULTADOS E DISCUSSÃO}

Todos os sujeitos deste estudo eram do sexo feminino com faixa etária entre 25 a 35 anos. Quatro delas possuíam pós-graduação lato sensu em Enfermagem Obstétrica e uma em Urgência e Emergência.

Estudos realizados em 2010 e 2016 dão conta de que mais de $90 \%$ dos profissionais de Enfermagem eram do sexo feminino. A feminização de funções vinculadas ao cuidar, principalmente na área da Enfermagem ainda vem sendo muito evidente (FERREIRA IP et al., 2010; RIBEIRO JF et al., 2016).

Identificou-se nas falas das entrevistadas que dentre os principais sinais e sintomas apresentados pelas gestantes, os mais comuns eram: presença de edemas nos membros inferiores, dor epigástrica, escotomas, e cefaleia na região da nuca, conforme destacado nos depoimentos de E1, E2, E3, E4 e E5:

“[...] A paciente tem uma cefaleia uma dor principalmente na nuca [...] vertigem, tontura, mas, assim, muitas pacientes são assintomáticas [...]" (E1)

"[...] Quando a paciente começar apresentar sinais de que tá começando a alterar a pressão não são todas, mas a maioria, ela já começa com edema nos pés, às vezes tem cefaleia, tem dor epigástrica, tem escotomas que já são sinais de iminências de convulsão [...]." (E2)

“[...] São alguns sinais clássicos como edema só em membro superior ou só em membro inferior a gente observa também a presença de um relato constante que são os escotomas. A paciente vem bem acima do peso e sobe a pressão e com isso vem a cefaleia que refere na região da nuca [...]." (E3)

"[...] Paciente vem bem acima do peso e com a cefaleia que refere na região da nuca." (E4)

"[...] Tem paciente que chega assintomática que relata uma dor de cabeça muito leve, um desconforto, apresenta sobrepeso, um mal-estar [...]" (E5) 
Conforme o Ministério da Saúde, dentre as manifestações clínicas predominantes do quadro destacam-se a elevação da pressão arterial, oligúria, repercussões cerebrais (cefaleia, torpor, obnubilação), visuais (escotomas, visão turva e/ou dupla e cegueira), dor epigástrica, cianose, elevação de células hepáticas, presença de proteinúria e em quadros críticos plaquetopenia (BRASIL, 2010).

Os sinais e sintomas relatados pelas enfermeiras são corroborados com outros estudos encontrados na literatura (MOURA MDR et al., 2011; LIMA EMA et al., 2010; CHRISTIAN A e KRUMWIEDE N, 2013). O enfermeiro deve estar apto a identificar os sinais e sintomas e realizar os primeiros atendimentos dessa gestante dentro e fora do ambiente hospitalar.

Autores ressaltam ainda que a tríade edema, proteinúria e hipertensão são os sinais clássicos da doença hipertensiva gestacional que levam a pré-eclâmpsia e, em casos mais graves, a convulsão característica do quadro (LIMA EMA et al., 2010).

Dentre os diversos fatores de risco indicados na literatura, destacam-se a diabetes mellitus, hipertensão arterial sistêmica, idade nos extremos da fase reprodutiva (gestação na adolescência ou depois dos 35 anos), histórico familiar ou pessoal de eclâmpsia, nefropatias, primiparidade, baixa escolaridade e obesidade (MOURA ERF et al., 2010).

O sobrepeso evidenciado pelas falas de E4 e E5 além de trazer consequências à gestante, também pode acarretar repercussões ao feto, como macrossomia fetal (MARQUES DSM et al., 2019).

A obesidade, caracterizada pela circunferência abdominal acima dos valores de referência é um fator preditivo para diversas patologias cardiovasculares. Um dos cuidados que devem ser realizados é a diminuição do peso, decorrente da redução da ingesta calórica, ocasionando diretamente a diminuição da pressão arterial, mecanismo explicado pela queda da insulinemia, redução da sensibilidade ao sódio e da atividade do sistema nervoso autônomo simpático (BRASIL, 2006).

O enfermeiro é o profissional que frequentemente encontra-se na linha de frente de atendimento às urgências e emergências obstétricas, sendo o primeiro a ter contato com a parturiente. Desta forma, é necessário que a assistência prestada por ele seja pautada em evidências científicas fortes e atualizadas. A anamnese detalhada, o exame físico cuidadoso e o constante acompanhamento dos níveis pressóricos são exemplos de cuidados demandados inicialmente à gestante e facilitam a detecção precoce de casos de préeclâmpsia (FERREIRA MBG et al., 2016).

Quando questionadas sobre quais os cuidados de Enfermagem comumente executados na maternidade, o cumprimento da prescrição medicamentosa e aferição dos sinais vitais, em especial pressão arterial, foram condutas lembradas pelas enfermeiras, de acordo com os depoimentos abaixo:

"[...] Primeiramente os cuidados de Enfermagem são realizados na admissão quando a paciente é admitida [...] realizamos as medicações [...] sulfato de magnésio na fase rápida, prevenível da eclâmpsia [...] depois a fase lenta efetuamos sondagem vesical para controle da diurese e sinais vitais, principalmente a PA (pressão arterial)" (E1)

“[...] Primeira coisa nos cuidados das pacientes com alteração na pressão é o controle rigoroso da pressão. Às vezes o médico coloca, dependendo da gravidade, de 2/2 horas de $4 / 4$ horas de $3 / 3$ horas de 6/6 horas a gente segue à risca [...] dependendo das queixas da paciente a gente também vê em horário menor. Primeira coisa é o controle rigoroso da pressão e avaliação dos sintomas e queixas das pacientes, se apresenta cefaleia, questão dos edemas, dor epigástrica se elas já chegam usando algum medicamento [...]" (E2)

"[...] Cuidado com a alimentação deve ser rigoroso, pois elas são bastante resistentes e difíceis, mesmo sendo ofertada a dieta da própria clínica, elas e os acompanhantes fazem uso de outros alimentos que não são prescritos pela nutricionista e a ausência do tratamento do uso medicamentoso, elas não dão muita importância [...]" (E3) 
"[...] Os cuidados de Enfermagem principalmente é cumprir à risca a prescrição médica. Essa paciente segue o protocolo tem que ser sulfatada, sondada, coletado os exames. Depois de monitorizadas, a gente tem que tá aferindo sinais vitais rigorosamente comunicando todas as intercorrências para o médico [...]" (E4)

"Temos todo um cuidado com essas pacientes, seguimos rigorosamente a prescrição médica, para que não venha evoluir para uma eclâmpsia, verificamos os sinais vitais de hora em hora, aplicações de medicamento, tudo isso conforme prescrição médica [...]" (E5)

Os cuidados realizados pelo enfermeiro na gestante com pré-eclâmpsia devem ser executados de maneira precisa e com atenção redobrada. São condutas comuns a aferição da pressão arterial quatro vezes ao dia, garantia de repouso no leito, avaliação de proteinúria, orientações para verificação materna diária dos movimentos fetais e observação dos sinais e sintomas das síndromes hipertensivas (FERREIRA IP et al., 2010).

Outros cuidados demandados relacionam-se à avaliação do local e extensão do edema, caso o apresente. Diariamente deve-se manter o registro preciso da ingestão de líquidos e da diurese, monitoramento dos sinais vitais, valores séricos e urinários de eletrólitos e proteínas, além de verificar os possíveis indicadores de sobrecarga/retenção de líquidos (LACERDA IC e MOREIRA TM, 2011).

Muito se falou dos cuidados clínicos ofertados, deixando-se de lado o suporte emocional que também deve ser oferecido pelo enfermeiro. Frequentemente, o diagnóstico de pré-eclâmpsia só é revelado à gestante no momento de necessidade de internação hospitalar em consequência da condição clínica grave ou pela iminência do parto. Sentimentos como surpresa, choque e ansiedade tomam conta da gestante no pósdiagnóstico.

Nesse sentido, é de competência do enfermeiro proporcionar uma comunicação dialógica com a parturiente e também com seu acompanhante acerca de alterações vividas nesse período, ofertando-lhes espaço para esclarecimento de dúvidas e verbalização de sentimentos, ideias preconcebidas sobre o quadro e preocupações a fim de facilitar o bom entendimento sobre seu tratamento e o motivo de sua permanência um pouco mais duradoura até a sua estabilização e liberação para o seu domicílio (MOURA ERF et al., 2010).

Atrelada ao diálogo é de fundamental importância a orientação à mulher sobre o plano terapêutico pósalta, de modo a prevenir agravos tardios das síndromes hipertensivas. $\mathrm{O}$ acompanhamento do pós-parto pela Atenção Básica em longo prazo, o controle de dados clínicos e o incentivo à adesão de hábitos de vida saudáveis são cuidados relevantes a serem dedicados (SILVA MLC et al., 2014).

Observou-se também nos discursos das entrevistadas ausência de cuidados relacionados à vitalidade fetal, priorizando-se apenas a monitorização da mãe, o que evidencia fragilidade da assistência realizada no quesito binômio mãe-filho. Além das condições maternas, o Ministério da Saúde recomenda que aspectos fetais também sejam avaliados, como: contagem diária dos movimentos fetais, investigação do crescimento fetal e do líquido amniótico, cardiotocografia basal e reavaliação materna-fetal em casos de mudanças clínicas abruptas (BRASIL, 2010).

\section{CONCLUSÃO}

A pré-eclâmpsia possui grande relevância científica ao interferir no processo da maternidade, romper a naturalidade da gestação e repercutir na vida do binômio mãe-filho. Conhecer as condutas de Enfermagem implementadas ao seu tratamento é essencial para a garantia de qualidade do atendimento ofertado. Salientase uma falha primária na assistência identificada neste estudo, em que muitas mulheres só descobrem a patologia no momento de internação, o que gera inúmeros desconfortos emocionais para a gestante que já passa por alterações hormonais que afetam seus sentimentos. Tal desconhecimento sobre a doença poderia ser sanado por meio de assistência pré-natal efetiva, com acompanhamento contínuo, tendo em vista que a assistência durante a gestação pautada em orientações e correção de dúvidas faz com que a gestante se envolva no processo de autocuidado, proporcionando seu protagonismo. 


\section{REFERÊNCIAS}

1. AGUIAR MIF et al. Sistematização da assistência de enfermagem a paciente com síndrome hipertensiva específica da gestação. Rev. Rene, 2010; 11(4): 66-75.

2. BRASIL. Ministério da Saúde. Secretaria de Atenção à Saúde. Departamento de Ações Programáticas Estratégicas. Gestação de alto risco. Manual Técnico / Secretaria de Atenção à Saúde, Departamento de Ações Programáticas Estratégicas. 5a ed. Brasília: Ministério da Saúde, 2010.

3. BRASIL. Secretaria de Atenção à Saúde. Hipertensão arterial sistêmica para o Sistema Único de Saúde. Cadernos de Atenção Básica, no 15. Brasília: Ministério da Saúde; 2006.

4. BRITO KKG et al. Prevalência das síndromes hipertensivas específicas da gestação (SHEG). Rev. Pesqui. Cuid. Fundam., 2015; 7(3):2717-2725.

5. CHRISTIAN A, KRUMWIEDE N. Simulation enhances self-efficacy in the management of preeclampsia and eclampsia in obstetrical staff nurses. Clin. Simul. Nurs., 2013;9(9):369-377.

6. FERREIRA GD et al. Insulin stimulation of Akt/PKB phosphorylation in the placenta of pre-eclampsia patients. Med J, 2013; 11(2):387-191.

7. FERREIRA IP et al. Gestantes: conhecendo os benefícios da prática de exercícios físicos em uma unidade de saúde da família. Semana Educa, 2010; 1(10):20-25.

8. FERRIRA MBG et al. Assistência de enfermagem a mulheres com pré-eclâmpsia e/ou eclâmpsia: revisão integrativa. Rev Esc Enferm USP, 2016; 50(2):324-334.

9. LACERDA IC, MOREIRA TM. Características Obstétricas de Mulheres Atendidas por Pré Eclâmpsia e Eclâmpsia. Acla Scientrarum Health Sciences, 2011; 33(1):71-76.

10. LIMA EMA et al. Conhecimento e atitudes dos enfermeiros diante de gestantes com sintomas da Doença Hipertensiva Específica da Gestação (DHEG) atendidas em Unidades Básicas de Saúde (UBS). J Health Sci Inst., 2010; 28(2):151153.

11. MARQUES DSM et al. A relação entre pré-eclâmpsia e obesidade: uma revisão integrativa. Cadernos da MedicinaUNIFESO, 2019; 2(2):56-62.

12. MASSUCATTI LA et al. Prevalência de diabetes gestacional em Unidades de Saúde Básica. Rev Enferm Atenção Saúde, 2012; 1(1):70-79.

13. MINAYO MCS. O desafio do conhecimento: pesquisa qualitativa em saúde. 14ª ed. São Paulo: Hucitec; 2014.

14. MOURA ERF et al. Fatores de risco para síndrome hipertensiva específica da gestação entre mulheres hospitalizadas com pré-eclâmpsia. Cogitare Enferm., 2010; 15(2):250-255.

15. MOURA MDR et al. Hipertensão Arterial na Gestação - importância do seguimento materno no desfecho neonatal. Comun. Ciênc. Saúde, 2011; 22(1):113-120.

16. OLIVEIRA ACM et al. Fatores Maternos e Resultados Perinatais Adversos em Portadoras de Pré-eclâmpsia em Maceió, Alagoas. Arq Bras Cardiol, 2016; ahead print, 0-0.

17. RIBEIRO JF et al. Contribuição do pré-natal para o parto normal na concepção do enfermeiro da estratégia saúde da família. R. Interd, 2016; 9(1):161-170.

18. SAMPAIO TAF et al. Cuidados de enfermagem prestados a mulheres com hipertensão gestacional e pré-eclâmpsia. Revista Saúde Física \& Mental, 2013; 2(1):36-45.

19. SILVA MLC et al. Mulheres com risco cardiovascular após pré-eclâmpsia: há seguimento no Sistema Único de Saúde? Rev Latino Am Enfermagem, 2014; 22(1):93-99.

20. SOUZA ALS et al. The high-risk pregnancy in view of nursing: a review study. Rev Pesqui Cuid Fundam, 2011; 4(1):1572-1581. 